\title{
Title: The Domain Relativity of Evolutionary Contingency
}

\begin{abstract}
A key issue in the philosophy of biology is evolutionary contingency, the degree to which evolutionary outcomes could have been different. Contingency is typically contrasted with evolutionary convergence, where different evolutionary pathways result in the same or similar outcomes. Convergences are given as evidence against the hypothesis that evolutionary outcomes are highly contingent. But the best available treatments of contingency do not, when read closely, produce the desired contrast with convergence. Rather, they produce a picture in which any degree of contingency is compatible with any degree of convergence. This is because convergence is the repeated production of a given outcome from different starting points, and contingency has been defined without reference to the size of the space of possible outcomes. In small spaces of possibilities, the production of repeated outcomes is almost assured. This paper presents a definition of contingency which includes this modal dimension in a way that does not reduce it to the binary notion of contingency found in standard modal logic. The result is a conception of contingency which properly contrasts with convergence, given some domain of possibilities and a measure defined over it. We should therefore not ask whether evolution is contingent or convergent simpliciter, but rather about the degree to which it is contingent or convergent in various domains, as measured in various ways.
\end{abstract}

Author: Cory Travers Lewis

Author's Affiliation: Institute for the History and Philosophy of Science and Technology, University of Toronto

E-Mail: cory.lewis@utoronto.ca

Address: 91 Charles Street West, Victoria College, Room 316, Toronto, Ontario M5S 1K7

Key Words: contingency, convergence, evolution, modality

Acknowledgments: This paper has been transformed several times over by excellent feedback. I am grateful for comments on a much earlier version from John Beatty and Eric Desjardins. Two quite different versions were presented to meetings of the Consortium for the History and Philosophy of Biology, both of which received helpful commentary and suggestions. Throughout the writing process I have received invaluable feedback from Denis Walsh and Philippe Huneman, as well as reading groups at the IHPST Toronto, and two anonymous reviewers. This research was also supported by funding from the Social Sciences and Humanities Research Council, and the Ontario Graduate

Scholarship.

\section{Introduction}

Stephen J. Gould (1989 : 45) famously claimed that if we were to run the tape of life all over again, we would see very different outcomes. Instead of birds, bees, trees, and people, radically different species 
with different arrangements of organs could have appeared from the same or nearly the same point of origin. Others disagree, claiming that the fact that evolution has often converged on the same or similar outcomes in widely different lineages shows that if the tape of life were run over again, the result would look much the same (Conway-Morris 2003, 2010). This is one of the most fundamental questions we can ask about life on Earth: how contingent are the outcomes we see?

Several definitions of contingency have been advanced with the tape-of-life question in mind, and the main business of this paper will be to critically analyze them, and present an improved alternative. The definitions I will look at are advanced by Beatty (2006), Ben-Menahem (1997), and Powell (2009). The aim of these definitions is to give precision to Gould's claim that evolutionary outcomes are somehow highly contingent. However, I will argue that when read carefully, these definitions fail to provide the desired contrast between contingency and convergence. Opponents of Gould's thesis, like Conway-Morris, argue that not only would evolution have ended up in largely the same places if run over again, but that in fact we can see cases where evolution did so, from dissimilar starting points. This, Conway-Morris claims, shows that evolution is predictable like any other natural phenomenon $(2003,2010)$. All of the authors considered here accept this way of framing the debate, opposing contingency and convergence, but their definitions fail to reflect this opposition. Taken strictly, their definitions of contingency are compatible with any degree of convergence.

I argue that this failure to produce the desired contrast between contingency and convergence results from trying to define contingency without reference to the domain(s) of biological possibilities which evolution explores. That is, they try to define contingency in terms of the occurrent properties of a causal process without making reference to its modal profile. This makes it impossible to connect contingency with convergence. Convergence is the repeated production of an evolutionary outcome from different starting points. But given a small space of possible outcomes, no matter whether a process is indeterministic (Beatty 2006), or simply sensitive to initial conditions (Ben-Manehem 1997; 
Powell 2009), the same outcomes will often come up. Without making reference to the size and structure of the domain of possibilities being explored (the modal profile of the process), neither indeterminism nor sensitivity to initial conditions meaningfully contrasts with the expectation of repeated outcomes.

Having demonstrated this difficulty, I then present my own definition of contingency which builds on these previous proposals, but includes the missing modal dimension. I argue that contingency should be thought of in terms of volumes ${ }^{1}$ in various domains of possibilities. Roughly, the degree to which something occupies a relatively small volume of a domain of possibilities is the degree to which it is contingent. More precisely, and drawing on Powell's insight that contingency should be judged relative to trajectories through a space of possibilities rather than states, evolutionary contingency is the degree to which a relatively constrained set of initial conditions results in a relatively large set of outcomes. What counts as 'constrained' or 'large' is defined relative to a domain, and a way of measuring that domain. In determining the degree of contingency of an evolutionary process it is the relative volume of the set of initial conditions and the set of outcomes which is considered, but these volumes are each defined in terms of the total domain in which they are embedded. Meaningful judgements about these volumes could likely be made informally, but I argue that to make claims about the contingency of an evolutionary process fully precise, the relevant domain must be explicitly defined and measured.

After presenting my proposal I describe a handful of such domains, like the space of possible morphologies, possible proteins, and possible ecological roles. These examples illustrate the complex ways in which well-informed judgements about contingency depend on our way of delineating and

\footnotetext{
${ }^{1}$ It is not strictly accurate to talk about 'volumes' in this context, since 'volume' implies a three dimensional space and many domains of possibilities include more than three dimensions. 'Hyper-volume' would be more accurate in cases with more than three dimensions (thanks to an anonymous reviewer for pointing this out). Generally, any reference in this paper to 'volume' in a space of possibilities should be regarded as an intuitive way of talking about measures, in the sense of measure theory, over the domain. For more detail on how measure theory can be applied to domains of possibilities, see Lewis and Belanger (2016).
} 
carving up a given domain. They therefore suggest that there will not be a single, simple answer to the question of whether evolutionary outcomes are contingent-they very much are contingent in some senses, and not very contingent at all in others. It could plausibly be the case, for example, that evolution is highly contingent in terms of the macromolecules it produces, but convergent in the space of possible body-plans. But in either case, it depends on how each specific domain is measured. So there is no single answer to questions like “How contingent is the evolution of the eye?" To make that question answerable, we must say whether we mean, for example, the molecular biology of eyes, their overall morphology, or their place in the life of a living thing. If this proposal is worthwhile, it is because it illuminates what we need to know in order to make well formulated judgements about contingency — we need to know what domain is under question, and how volumes in it are best measured.

I begin by looking at John Beatty's attempt to make Gould's somewhat intuitive picture philosophically precise. Beatty offers up two senses of contingency, and along the way I consider the alternate proposals of Ben-Manehem and Powell. After showing why each proposal requires the addition of a more robust modal component, I then present my own definition, which I show solves the issues identified with the best current proposals. Essentially, evolutionary contingency must be defined in a domain-relative way. I then present a number of examples of biological domains of possibilities, in which evolution could be contingent or non-contingent. One upshot of the discussion is that evolution could be highly contingent in some domains (e.g., the domain of possible proteins) while being highly convergent in others (e.g., the domain of overall body-plans). Getting precise about the stakes of the contingency/convergence debate therefore forces us to separate the overall thesis that evolution is contingent into a variety of more restricted, domain-specific theses.

Another consequence of thinking about contingency in domain relative terms is the process by which domains are defined and measured comes to the foreground. Some natural questions arise in this 
context: how exactly are these domains identified, and how are volumes assigned to them? These questions are structurally very similar to the question of how probability spaces are defined and measured. How do we decide which sample space to define a probability measure over, and how to define the measure itself? Very likely there is not a general algorithm for doing so, no completely general solution to the so-called 'reference class problem’ (Hajek 2007). Nonetheless, looking at biologists working on defining domains of possibilities and measuring contingency in those domains gives us examples of how this problem is tackled in specific empirical contexts. I will argue that it is the empirical context of a given investigation that provides reasons to define a domain in one way rather than another. For example, in the case of the domain of possible proteins, it is the mechanism of protein synthesis that lays out the appropriate boundaries of the domain, and our specific research interests which determine what measure should be defined over that domain. If this is correct, then biologists are sometimes engaged in what Roca-Royes (2007) calls modal empiricism - the investigation of modal facts by empirical methods. If my examples are valid, biologists are already well engaged in the domain-relative analysis of contingency in evolution, so will not gain much from having philosophers confirm their approach. However philosophers, I claim, can learn an important lesson about how modal facts are generated via empirical research from this domain-relative approach to evolutionary contingency.

\section{Two Types of Contingency}

John Beatty (2006) has looked carefully at the issue of contingency in evolution as Gould formulated it, and found at least two distinct senses of contingency at play. This distinction will remain part of my amended proposal, so it will be worthwhile focusing on it at the outset. Before specifying this distinction in more precise (and I will argue, ultimately unhelpful) terms, Beatty (2006) presents an intuitive gloss on it which is very helpful: he distinguishes contingency per se, from contingency upon. 
Contingency per se describes something which is neither impossible nor necessary. So something is contingent per se just in case it could have been otherwise. Beatty tries to make the concept more precise by interpreting it in terms of indeterminism. Something is contingent per se, on his reading, if a different outcome could have come about from identical initial conditions. This feature of evolutionary processes, that they seem chancy and indeterministic, was a major feature of Beatty's (1995) argument for the Evolutionary Contingency Thesis (ECT). Since the very processes which produces evolutionary outcomes is chancy, he argued, those outcomes could have been otherwise. But what is needed in this context is a graded notion of contingency, so that we can make sense of the very common practice of describing some outcomes as 'highly' contingent ${ }^{2}$. I will say more on this point shortly.

The other important type of contingency which Beatty identifies in Gould's argument is contingency upon. This is the sort of contingency which is in play in a sentence like "Renewal of the contract is contingent upon meeting our sales targets.” $\mathrm{X}$ is contingent upon $\mathrm{Y}$ if the bringing about of Y makes X more likely than the absence of $\mathrm{Y}$. This sort of contingency is constantly in play in evolution, where past adaptations create both opportunities for and constraints upon the future paths which evolution can take. This, I take it, is the main point of Gould and Lewontin's (1979) famous argument against explaining every feature of organisms as adaptations. Sometimes, the directions that evolution can take are heavily constrained or enabled by (contingent upon) what has happened in the past. Beatty tries to make this notion more precise by describing it in terms of causal dependence, the dependence of current states of a system on past states.

Reaching towards a unified treatment, Beatty combines these two sorts of conditions: a process is contingent just in case its past states are causally necessary, but insufficient to bring about some

2 It is worth noting that Beatty (1995 : 228-229) explicitly flags the need for a concept of contingency which comes in degrees, and warns that his account as stated may be too simple in that regard. 
future state. Stated as such however, this definition of contingency seems to be trivially satisfied by practically any less-than-strictly-deterministic causal process. Turner (2011) makes this point vivid:

Consider, for example, the sequence of events involved in preparing a pasta dish. Filling the pot was necessary, but not sufficient, for boiling water on the stove. [...] Boiling the water on the stove is necessary, but not sufficient, for cooking the noodles. Cooking the noodles is necessary, but not sufficient, for producing fettucini alfredo. (169)

In fact, it seems hard to imagine a non-deterministic causal process which would not satisfy Beatty's definition of contingency as stated. But as I will argue below, even deterministic causal processes can be contingent in an important sense, so this combined definition seems to miss the point. More importantly, I will argue in the remainder of this section that this way of treating contingency does not allow us to contrast contingency with the likelihood of finding convergent evolutionary outcomes, which was the point of clarifying contingency in the first place.

Let us begin with contingency per se. Beatty (2006) defines it in terms of causal insufficiencythe possibility of the different outcomes resulting from the very same starting point. Can it be the case that a process is indeterministic in this way, yet reliably produces the same or very similar outcomes? Yes, it seems clear that this will be the case given a sufficiently small domain of possibilities. For example, if we roll a six sided die 1000 times, the same numbers will come up very often indeed. Even if we assume that the underlying physics of rolling a die is indeterministic in a metaphysically deep sense, the fact that there are only six outcomes guarantees that given enough trials, we will see the same outcomes over and over again. Of course, the domain of evolutionary outcomes is presumably much larger, so we do not have the same a priori guarantee of repeated outcomes. But the point here is that the fact that a process is indeterministic tells us nothing about how many possible outcomes could result from it. . That means that Beatty's definition of contingency per se does not provide the wanted contrast with the expectation of evolutionary convergence.

It may seem that the trouble here is indeterminism, but the same problems arise in treatments 
that are phrased specifically to avoid that assumption. Consider Powell's (2009, cf. 2012) argument that evolutionary contingency should be defined in terms of similar—rather than identical—starting points leading to radically different outcomes. Beatty's treatment, he claims, needs to assume that the world is indeterministic in a deep metaphysical way, a point which should not be relevant in this debate. If the world is strictly deterministic, then running the tape of life over again will necessarily produce exactly the same result, and the thought experiment does not work. Powell tries to refine this unpredictablity condition into something that does not have this odd dependence on seemingly irrelevant (and probably undecidable) metaphysical issues. He employs the notion that small change to initial conditions can explode to large final differences:

Broadly construed, radical contingency is the notion that arbitrarily small differences in input variables produce disproportionately great disparities in outcomes. Thus, outcome $O$ in system $S$ is radically contingent iff a marginal change in some initial condition $I^{1} . . I^{n}$ of $S$ would tend to result in outcome $O^{*}$ where $O *$ is radically disparate from $O$. The key point is this: Marginal disparities in initial conditions tend to lead not only to different — but radically differentevolutionary outcomes. (2009 : 394, emphasis in original)

A very similar treatment of contingency had been advanced by Ben-Menahem (1997), who argues “contingency [...] increases in direct [...] proportion to sensitivity to initial conditions” (99) This has the nice effect of avoiding the tricky determinism versus indeterminism debate, since now we have a way of describing divergence of nearby, rather than identical, starting points. ${ }^{3}$ It is also compatible with indeterminism, since identical starting points are also, of course, similar. This sets up contingency as in opposition to equilibrium outcomes, a notion which Beatty has endorsed elsewhere (Beatty and Desjardins 2009). The idea is that some processes go to equilibrium, which means that very different initial conditions will end up at the same result. No matter where I place a drop of black ink in a glass

\footnotetext{
${ }^{3}$ It may be helpful to distinguish here between sensitivity to initial conditions from chaotic dynamics. Sensitivity to initial conditions is a necessary feature of chaotic dynamics, but is not generally regarded as a sufficient condition. The exact definition of chaotic dynamics is a matter of ongoing debate, but candidates for additional necessary conditions include determinism (Smith 2007), complex non-periodic orbits in state space (Hunt and Ott 2015), and a tendency to both diverge sensitively and converge towards so called 'strange attractors' (Batterman 1993). None of these features are strictly contained within the idea of sensitivity to initial conditions, since it is compatible with both determinism and indeterminism, and does not strictly require that the system be confined to orbiting an attractor.
} 
of clear water, it will fairly soon end up mixed throughout, which is its equilibrium point. It is to that extent non-contingent what happens when ink is dropped in water, on the above definition, because the outcome of interest is very insensitive to small changes in initial conditions. We can tell that ink was dropped in the water - this information is not destroyed. However, where exactly the ink was dropped, on this side of glass or that, will be unrecoverable once it has dispersed. This explicitly connects contingency with the degree to which something is "counterfactually robust” (Powell 2009 : 395). As far as it goes, this part of Powell's proposal seems exactly right.

However, this formulation suffers from the same disconnect from convergence (or lack of convergence) as Beatty's 'unpredictability' sense of contingency: even if outcomes can differ "radically" from their initial conditions, if the total space of possible outcomes is small then the same outcomes will turn up again and again. If we divide the space of initial conditions of a coin-flip into two, and the space of outcomes into two, there should be practically no correlation between initial conditions and outcomes. The outcome can differ as 'radically' as possible given this domain. But in the case of such a restricted set of possibilities, any given outcome, heads or tails, seems only minimally contingent. Far less contingent, say, then that I should have ended up with the exact set of protein-coding genes that I did. In this comparison the outcome with much stronger connections between initial conditions and outcomes, my exact genetic code, has the greater contingency simply because it occupies such a small volume of a vast space. Powell's proposal is an improvement in that it eliminates the dependence on an assumption of indeterminism, and begins to frame contingency counterfactually. But without being supplemented by explicit reference to the domain of possibilities being explored, it still cannot provide the desired contrast with convergence.

This same basic issue, in a slightly different form, plagues Beatty's treatment of contingency upon. Can it be the case that the state of a system can depend upon its past states (causal dependency) and yet produce the same outcome repeatedly? It seems so. The orbit of the earth around the sun was 
produced by a historical process, and its details (like the velocity of earth, and the shape of its ellipse) depend heavily on its past states. But the sun rises every morning, in a quite predictable fashion. So causal dependence, as such, does not seem to provide any contrast with convergence either. A system can depend heavily on its past states, yet produce the same outcomes over and over.

In fact, the state of every process which is less than totally random depends on its past states, so this condition appears on its face to be trivial. But the sort of contrast Beatty has in mind here is not, we should note, totally random processes. He is trying to distinguish between what Sober (1988) called information preserving and information destroying processes. A system is information preserving just in case the particular past states of a system continue to be preserved in the way it unfolds into the future. This property of being information preserving is central to the accounts of historicity (a concept related to but importantly different from contingency) proposed by Beatty and Desjardins (2009) and Desjardins (2009, 2011). They trace the lineage of this idea back to Lewontin, who opposed historicity with going to equilibrium (Lewontin 1974 : 269).

To rephrase in Sober's language, to the extent that a system reaches one global equilibrium point, it is information destroying. To the extent that it does not, it may be information preserving. That is, we can tell something about the past states of a system by which local equilibrium it ends up in. The important insight here is that local equilibria are not necessarily opposed to historicity. A process like evolution can be both optimizing, as the adaptationists long argued (Dawkins 1976 ; Maynard Smith 1978 ; Dennett 1995) and still be information preserving. When a ball rolls from the rim of a bowl to the bottom, its ending up at the bottom does not hinge on precisely where it started on the rim. It is this sense in which the past states of the system do not matter - any one of a range of possible starting points results in the same ending point. Of course the past states of such a system are relevant to how the ball ends up at the bottom of the bowl - there has to be a ball, and a bowl, and the bowl has to be right side up, and the ball has to be on the rim, and not fall outside of the bowl, and so on. Contingent 
upon all of these conditions being met, the result is independent of where the ball starts on the rim. This provides the contrast Beatty is trying to make, between systems which depend on the initial conditions being just so in order for an outcome to obtain, and systems in which the outcome would obtain across a broader range of variables. My claim is that it is the distinction between local and global equilibria doing the work in distinguishing the degrees to which something can be contingent. To the degree that a system's possible trajectories through its state space all fall into one global equilibrium, it is noncontingent. If there are many local equilibria it can occupy, it is contingent, since there are many different outcomes that could obtain. But to make this distinction we need the concept of volume in a space of possibilities, to articulate the difference between local and global equilibria. The only difference between the two is that global equilibria dominate a space of possibilities, and local equilibria are relatively small.

That means that what is missing from this account of contingency upon is the distinction between systems in which past conditions must be very specific (occupy a small volume of a domain of possibilities) for an outcome to obtain, and systems in which a broad range of past states could bring about the same outcome. That is, the same dimension is missing from this way of treating contingency as was missing from Beatty's account of contingency per se: the modal dimension. The distinction between local and global equilibria, which must be included to make contingency upon non-trivial, is a distinction in terms of the volume of these equilibria in some space of possibilities. So once again, we cannot produce the wanted contrast between contingency and convergence without including some concept of volumes in domains of possibilities. In the next section, I proceed to try to produce a way of treating these two types of contingency which includes this modal dimension, and therefore generates a meaningful contrast with convergence.

\section{A Graded Modal Treatment of Contingency}


If the above is correct, the available definitions of contingency fail to produce a contrast with evolutionary convergence because they make no reference to the size (or more accurately, the measure) of the space of evolutionary outcomes. To remedy this, I propose the following picture, which incorporates insights from both Beatty and Powell's accounts. Begin with a set of initial conditions, and a set of outcomes, which occupy the same domain. The outcomes are contingent to the degree that they occupy a large measure of the domain, relative to the measure of the set of initial conditions. This picture incorporates Powell's view that both initial conditions and outcomes are relevant to determining the contingency of a set of trajectories through the domain. This is important, because the contingency of an outcome varies dramatically depending on what we consider the initial conditions to be. That humans have our current genetic structure, for example, may be highly contingent with respect to the initial conditions of life several billion years ago, but not very contingent at all relative to one generation ago. Similarly, the degree of contingency we assign to an outcome will vary depending on whether we want to know what would have happened from a single initial condition, or whether we want to consider a range of possible initial conditions. For example, the question of whether life on earth, starting from our actual past, could have turned out otherwise, is a rather different one than whether life anywhere in the universe will converge on the same forms. The strongest form of the antiECT put forward by Conway-Morris (2003) suggests that life anywhere in the universe would converge on human-like forms. It is useful to be able to distinguish this from the thesis that given the very specific conditions under which life evolved on earth, some forms are more likely than others. This formulation also captures the distinction that Beatty makes between contingency per se and contingency upon. What we want to know, I propose, is how contingent per se a set of outcomes is, contingent upon a set of initial conditions. Incorporating Powell's insight that we must consider both outcomes and initial conditions prevents the combination of these two forms of contingency from becoming trivial, and relativizing both to a domain of possibilities generates the desired contrast 
between contingency and convergence.

The point that the significance of convergences depends on how we measure a space has been made by other authors (e.g., Huneman 2010). Particularly relevant here are the insights of Powell and Mariscal (2015). They argue that convergences must be sorted along several axes: specificity (meaning roughly degree of abstraction), independence (the degree to which convergences rely on shared developmental resources) and scope (the range of conditions under which a convergence is to be found). The relevance of a convergence to evaluating the ECT must, they claim, be understood relative to where it falls along these three axes. A highly non-specific, non-independent, and narrow scope convergence makes very poor evidence against the ECT.

This, I take it, is all correct. In the language of this paper, we could say they are arguing that it matters how we measure the domain of evolutionary outcomes. The additional insight that thinking in terms of medium-strength modality adds to this discussion is the point that it matters which domain of possibilities we are talking about, and that there may be many such domains. So rather than one overall ECT that various convergences may tell against, we should recognize many separate ECTs, each corresponding to independent domains of biological possibilities. It also matters how we measure a given domain. Many different measures can be defined over a single domain, resulting in different assessments of how 'large' a given set of outcomes or initial conditions will be.

Both of these points, domain-relativity and the measure-relativity that follows from it, raise serious questions. How are domains identified, and how are they measured? Are domains objective features of reality, or merely human constructs? These are very large scale questions about the metaphysics and epistemology of modality, which are unlikely to be resolved in a single go. However, if the above arguments are sound, we are forced to confront them if we wish to have a fully articulated picture of evolutionary contingency. The next section looks at some case studies in order to try to glean some insight into how modality features in biological practice. 


\section{Domain Relativity}

In this section, I look at two case studies in identifying and measuring domains of possibilities, the protein universe and morphospace. The overall lesson I wish to draw from them is that these spaces are defined and measured through empirical investigation. What researchers look to discover, I suggest, is a way of representing the domain of possibilities which assigns equal measures to equally possible outcomes. This is an application of the principle of indifference, a foundational principle in probability theory. Strevens (2013) argues for the careful separation of two versions of the principle of indifference however, an epistemic and a physical version. The epistemic version says: assign equal probabilities to outcomes that seem equally likely given your state of knowledge. The physical version says: assign equal probabilities to equally likely outcomes. He calls the application of this physical version of the principle equidynamic reasoning (36). Looking at the way biologists try to represent sets of biological possibilities reveals that they are employing equidynamic reasoning, trying to fit their representations to the modal structure of reality. They fix on biologically relevant possibilities, and attempt to measure those domains in accordance with this physical principle of indifference. This, I claim, is an instance of modal empiricism (Roca-Royes 2007), the discovery of modal facts through empirical methods.

However, readers with qualms about de re modal claims can make use of the framework presented here without subscribing to realism. For constructivists about modality, Strevens' epistemic version of the principle of indifference can fill in for the physical version I will adopt below.

\section{The Protein Universe}

The protein universe is the set of all possible proteins. It is a biologically relevant domain of possibilities, because of course proteins make up a great deal of living things, and a central product of gene transcription. Proteins are made up of amino acids arranged in a linear chain, so the protein 
universe consists of all of the possible linear chains of amino acids. That is to say, this particular domain of possibilities is picked out as the biologically relevant one by the mechanism of protein synthesis. If ribosomes were capable of inserting other molecules into these linear chains, or to assemble branching or looping structures of amino acids, then a different domain would be biologically relevant. Because of the (empirically discovered) facts of our molecular biology, the set of possible linear chains of amino acids is a highly relevant domain to consider.

The question of the contingency of evolutionary outcomes in this domain depends not just on the limits of the domain, but also crucially on how it is measured. There has been considerable debate about which are the best ways of measuring the domain, a debate which can be understood as an attempt to satisfy the demands of equidynamic reasoning.

The space of all possible amino acid sequences is impressive in scale and complexity. One can calculate the number of types of protein by raising the number of different amino acid types to the power of the length of the chain. There are types of 20 amino acids involved in life on Earth, and proteins range in length from peptides, which are only a few amino acids long, to huge repetitive proteins in human muscle called Titin, which can be ten thousand amino acids long (Trinick, 1994). If we take 200 amino acids as a very conservative upper bound, we get a figure of $20^{200}$, or $10^{260}$ different possible protein types.

If we simply define a measure over this space which treats all different amino acid sequences as totally separate proteins, it seems likely that a given protein type will be highly contingent relative to the broad history of life. But given that similar protein sequences will very often have similar biological properties, there is reason to believe that a totally indifferent measure over the domain is not the biologically relevant measure. There are a variety of proposals for ways to carve up the protein 
universe into regions and neighbourhoods. Forget worrying about each and every possible substitution of amino acids, and instead think of broad classes of proteins. For example, proteins fold themselves into various shapes, and it is very tempting to group proteins simply by these folds. This is a structural approach to protein classification. Characterizing protein structure in terms of folds is appealing, because the number of unique folds seems to be relatively small. Koonin et al. (2002) estimate that there are "probably no more than 10,000 folds in existence". That is quite a few relative to the number of chemical elements, but easily manageable compared to the number of possible sequences.

Alternately, Taylor (2002) proposes characterizing proteins in terms of their overall structure, and is bold enough to describe his formal treatment of that problem as a "periodic table" of protein structures. His treatment considers structural features smaller than a fold, and is meant to capture some of the structural detail that would be tossed out by just putting proteins into categories based on which major folds appear in them. Both proposals offer means of carving up the protein universe into neighbourhoods, and given such a method of subdivision we can then ask questions about how much of the space is occupied. It could be the case, for example, that although the space of sequences is barely explored, the space of folds is well explored. If folds are the most important aspect of proteins, then if the space of folds is well explored, we should say that the ECT with respect to the protein universe is false - life has at least touched most or all relevant neighbourhoods in the domain. By 'important' or 'relevant', I mean here relevant to the project of enforcing equidynamic reasoning.

But structural information does not exhaust everything that we want to know about proteins. We are also interested in their functional properties, meaning their capacity to perform biological functions like catalyzing reactions or binding to receptors (e.g., Brun et al. 2003; Rost et al. 2003). No fully general account of how the structural features of proteins translate into their functional properties is currently available, but the investigation is underway. For example, the enormous protein sequence 
database UniProt correlates information about the amino acid sequence of a protein, which tells us about its structure, with functional information gleaned from various empirical investigations. But so far the fit between the two is not tight: Pandey et al. (2006) review the literature on the structure/function connection, and find that so far "the structure-function correlation is not strong enough to enable the inference of function directly from a protein's structure” (32)

Nonetheless, some ways of categorizing the protein universe also have a mixed structural/functional character. Some argue that the proper way of dividing the protein universe is into domains, which are building-block like protein segments. The same domain can typically be found in many different protein types (Koonin et al. 2002). This mixed sequence/structure/function characterization of protein domains perhaps makes them appropriate for categorizing proteins, since our interests are presumably both in their intrinsic structural properties, their biological role, and also their evolutionary relationships. A mixed structural/functional way of categorizing proteins would generate yet another ECT, specific to this way of structuring the space.

Some authors have treated this question directly, arguing that claims of contingency in the protein universe are overblown, because we have naively taken sequence space to be the relevant way of decomposing the domain. Consider the argument made by Dryden et al. (2008), that contrary to the claim that the protein universe is unexplorably vast, life on Earth actually has a good chance of exploring most of the possible proteins. This of course would not mean that life could generate every possible sequence, since their number is indeed too vast. But that does not matter, because, as they argue, "the actual identity of most of the amino acids in a protein is irrelevant.” (953) If most substitutions of different amino acids into a given protein make no difference to its secondary or tertiary structure, or its function, should we really consider these irrelevant substitutions unexplored territory? Dryden et al. think not. 
The astronomically large estimate of the size of the protein universe I provided at the beginning of this section was based on the idea that there are 20 types of amino acids that go into terrestrial life. But Dryden et al. suggest that many of these amino acids will be functionally equivalent in many contexts. The extreme limit of this sort of generalization is to regard amino acids simply as presenting polar or non-polar surfaces when bonded in a chain, giving only two types (Dill 1999). Assuming a limit of 100 residues per protein, this gives us a space of $2^{100}$ or about $10^{30}$ different types. This radically reduces the number of protein types that need to be considered, bringing it much closer to the total number of proteins that may have been produced here on Earth. Dryden et al. put the upper limit on how many protein types life could have theoretically produced since it began 4 billion years ago at 4 $\mathrm{x} 10^{43}$ (though that is certainly meant as an upper bound rather than an estimate of the actual number). This means that if we simply carve the protein universe up a bit more roughly, it looks as though terrestrial life has had the chance to explore quite a bit of it.

The lesson here is simply that what biologists are up to when they define measures over the protein universe is trying to enforce equidynamic reasoning - two outcomes which are biologically distinct should occupy a greater measure of the space than two outcomes which are biologically indistinguishable. How exactly to measure the protein universe is then the question of which outcomes are biologically different enough to count as 'different'. This question is currently an open one, as we learn more about the structure of proteins and their functional roles in living things. It may be the case that different empirical questions make different measures more salient, so that there is not just one correct way to measure the domain. However this is clearly not to say that these measures are simply arbitrary, or mere human constructs. Biologists are attempting to identify those measures over the domain that best reflect the actual biological possibilities that are relevant in a given empirical context. 


\section{Morphospace}

Morphospace is the space of possible forms or body-plans. A variety of different molecules could make up a given form, and a given form could occupy a variety of ecological niches. This means that, conceivably, life could be very contingent in the protein universe (under some measure), but highly convergent in morphospace, or vice versa. And as with the protein universe, morphospace does not admit of a single straightforward measure. But unlike the protein universe, the most practical approach to morphospace seems to be to treat it as many separate domains, rather than to define many measures over the same domain. Maclaurin and Sterelny (2008) argue that a global morphospace is practically and theoretically intractable, because morphospaces are usually built to test for some adaptive mechanism or other. In practice biologists do not even attempt to define global morphospace, preferring to focus on more local spaces, like the space of coiled conical shells (Raup and Michelson 1965; Raup 1966, 1967) and the space of possible interior skeletons (Thomas and Reif 1993). These more local morphospaces are typically defined relative to some empirical question or other. And without such constraints, it becomes unclear how to connect and compare the apparently incommensurable dimensions, or to pick which dimensions should be included in the first place. Maclaurin and Sterelny $(2008,82)$ write: “Unless we have a specific clade and explanatory project in mind, there is no principled answer to the question "What are the dimensions of morphospace?"”,

So while morphological convergence is a rich source of evidence against local ECTs, only a comprehensive survey of the sub-domains of morphospace could falsify a more global morphological ECT, assuming such a thing could be formulated. In the examples below, it will be clear that the actual research done in theoretical morphology is all framed in terms of local spaces of possibility, with no apparent need to refer to a global morphospace. Belief in such a global morphospace is necessary if we are to defend even a morphologically global ECT, but theoretical morphology seems to be proceeding 
fine without it.

As an example of a local morphospace, consider Raup's (1966) landmark model of the space of coiled shells. It set a new bar for rigor in the tradition of defining quantitative variables over the form of organisms. A space of shells in four dimensions: "the shape of the generating curve (S), the whorl expansion rate (W), the position of the generating curve in relation to the axis (D), and the rate of whorl translation (T).” (1179) The idea is that a "generating curve”, which in the simplest case is a circle, rotates around a central axis. As it travels, it traces out the walls of a shell. It also expands at some rate $\mathrm{W}$, and moves along the axis at a rate $\mathrm{T}$. High values for $\mathrm{W}$ produce shells that rapidly expand before many degrees of rotation. High T values produce helicoid forms, which spiral away from their origin along the axis of rotation. And high D means that the generating curve moves away from the axis of rotation (the coiling axis) as the shell expands, rather than staying in contact with it, "as in many gastropods” (1181).

This produces a space of possibilities which Raup is happy to admit is very simple relative to reality. For example, he assumes that one of his variables, $\mathrm{S}$, is constant, because of the complexities involved in treating it as variable. It also excludes many dimensions that we may find relevant, like types of surface ornamentation. But these four dimensions (three really) are enough, Raup claims, to get going on the business of considering the relationships between the abstract space of possibilities, the distribution of actual organisms in it, and the functional constraints that organisms experience in a given environment.

We can therefore formulate a very local ECT which ranges over the domain of coiled-shell forms. According to Raup, quite a bit of the space is occupied by past or living species, so a radical ECT does not seem to be well supported. But at least on Raup's original formulation, the space is not 
completely occupied by actual organisms. There are gaps. On the question of these unrealized forms, Raup demurs on whether empty points in the morphospace represent "physiologically impossible shell forms or has the evolution of these taxa simply not had sufficient time in which to populate the entire block? [...] The answers to these and similar questions are necessary if we are to understand fully the evolution of coiled organisms.”(1185) So he is agnostic about whether life has explored this space completely or not, and therefore about whether unrealized forms are just inherently maladaptive, or if they simply have not yet been tried. But at least in a local context like this, with a specific measure over a specific domain, the question is precise enough to be meaningfully investigated ${ }^{4}$

A great many other local morphospaces have been investigated by biologists, and local ECTs could be defined over any of them. For example, Thomas and Reif (1993) define a 'skeleton space', a space of possible skeletal structures. But even given such a space, we face the problem of defining some specific measure over it, in order to assign volumes to the various neighborhoods. How to do so many not always be clear. As Maclaurin and Sterelny $(2008,68)$ put it:

It makes sense to think of a physical object being "as wide as it is deep” because width and depth can be measured in the same units. In contrast, most of the dimensions of morphospaces are only distantly related to our familiar three spatial dimensions, and their dimensions are rarely commensurable. Usually, the relationship between the dimensions is more like the relationship between the standard spatial dimensions and time. Many of the "distances” in such spaces are not even magnitudes.

Given some specific empirical question to answer, we may be able to specify how dimensions of morphospace should be related to each other. Certainly that is not conceptually impossible. But until we do so, there may not be a concrete answer to questions about the volume of morphospace occupied by actual life. The consequence for even a local ECT defined over some specific morphospace is that

\footnotetext{
${ }^{4}$ Raup's work was not the final word on defining the space of possible coiled shells. McGhee(1999, 77-140) reviews further refinements of the concept of the space of coiled shells, including the more general spaces defined by Okamoto (1988) and Ackerly (1989).
} 
there may not be a determinate answer to how contingent terrestrial life is with respect to that domain. So it may be the case that some ECTs are not evaluable.

But if the above is correct, we now at least have in hand the conditions that must obtain in order for a given ECT to have a determinate answer: we must be able to specify which domain we're talking about, and how to measure it. Given those two pieces of information, we are conceptually equipped to formulate a precise ECT and say what evidence would tell for or against it, to what degree. This does not entail that we have the relevant information to actually answer the question, of course, but at that point philosophy must give way to empirical science.

In the case of morphospaces, which domains and measures are relevant seems to be a function of the developmental processes which produce the variation on which evolution acts. Raup's space of coiled shells follows this pattern: the "generating curve" is the mechanism, and variations in its activity generate the phenotypic variation upon which selection acts. Evolutionary developmental biology fits this pattern, with its emphasis on those possibilities that are afforded by the developmental resources that are shared amongst so much of complex life on earth (Hall 2003). If developmental constraints make some phenotypes much more likely to arise, then broadly defined morphospaces like Dennett’s(1995) ‘design space’ (which includes all physically possible phenotypes) may be biologically less relevant than the space of phenotypes which can be reached by variations in development.

Again, the point here is that biologists seem to choose which domains and measures are relevant by employing equidynamic reasoning. Given an empirical research project, they attempt to represent the possibilities in a way that assigns equal measures to equally possible outcomes. Variations in research goals may make different domains and measures relevant, in the same way that different probability distributions may be assigned to an event given different reference classes. But nonetheless, 
the goal is always to match our representations to reality, to represent real features of the modal structure of the world.

Examples of this type of reasoning could be multiplied. Biologists also consider the space of possible ecological niches, sometimes called ecospace ${ }^{5}$. Pianka (1978) attempted to define a "periodic table of niches” (267), a space of possible ecological roles. He divided it in terms of r-selected and kselected organisms, as well as in terms of primary producers, herbivores and carnivores. Bambach (1983, 1985) likewise attempted to define a space of possible ecological roles, using slightly different dimensions, and Bambach et al. (2007) define a space of ecological roles for marine life. Similarly, astrobiologists debate whether life could have evolved using fundamentally different chemistry given different planetary conditions (Bains 2004). This, I would suggest, can be read as a debate about the space of possible chemistries that can support life.

\section{Conclusion}

I have argued for a graded and domain-relative conception of evolutionary contingency. The main motivation for this conception was to resolve the apparent failure of previous definitions of contingency to properly contrast with convergence, as the debate has assumed it should. Without mentioning the space of possibilities being explored, attempts to define contingency in terms of indeterminism or sensitivity to initial conditions does not produced the desired contrast with the likelihood of repeated outcomes. But once we do mention the domain of possibilities being explored, it becomes clear that evolutionary contingency is not a unitary fact about the history of life on Earth. Relative to one domain, it could be quite contingent, and at the same time, highly non-contingent in others. This separates the ECT into a multitude of more local theses about specific domains, measured

5 See McGhee (2011) for an overview. 
in specific ways. While this complicates the discussion considerably, removing any hope of definitively evaluating "the" ECT, it also brings us much closer to a position where we can meaningfully bring the available empirical evidence to bear on the now separated questions.

This proposal raises a number of questions, particularly with regard to how domains are identified as relevant and measured. I have argued that these are questions that are answered within specific empirical contexts, according to various research goals. But the guiding principle in these deliberations is the attempt to enforce equidynamic reasoning, to assign equal measures to equally likely outcomes. Outcomes which are not biologically distinct (according to the standards of a specific research program) can be treated as 'the same', and measures are defined accordingly. Since this conclusion is derived from looking at biological practice, it is unlikely to alter the way biologists proceed. However, it does help to clarify the conceptual structure of questions around the ECT.

\section{References}

Ackerly SC (1989) Shell coiling in gastropods: analysis by stereographic projection. Palaios 4:374-378 Bains W (2004) Many chemistries could be used to build living systems. Astrobiology 4:137-167 Bambach R (1983) Ecospace utilization and guilds in marine communities through the Phanerozoic. In: M. Tevesz \& P. McCall (eds) Biotic interactions in Recent and fossil benthic communities. Plenum Press, New York, pp 719-746

Bambach R (1985) Classes and adaptive variety: the ecology of diversification in marine faunas through the Phanerozoic. In: J. Valantine (ed) Phanerozoic diversity patterns: profiles in macroevolution. Princeton University Press, Princeton, NJ, pp 191-253.

Bambach R, Erwin, D (2007) Autoecology and the Filling of Ecospace: Key metazoan radiations. Palaeontology 50:1-22

Batterman R (1993) Defining chaos. Philos Sci 60(1):43-66

Beatty J (1995) The evolutionary contingency thesis. In: G. Wolters, J. Lennox (eds) Concepts, Theories, and Rationality in the Biological Sciences. University of Pittsburgh Press, Pittsburg, pp 4581

Beatty J (2006) Replaying Life's Tape. J Phil 103(7):336-362

Beatty J, Desjardins E (2009) Natural selection and history. Biol Philos 24:231-246

Ben-Menahem Y (1997) Historical Contingency. Ratio 10:99-107

Brun C, Chevenet F, Martin D, Wojcik J, Guénoche A, Jacq B, et al. (2004) Functional classification of proteins for the prediction of cellular function from a protein-protein interaction network. Genome biol 5:R6-R6

Conway-Morris S (2003) Life's Solution: inevitable humans in a lonely universe. Cambridge University Press, Cambridge Conway-Morris S (2010) Evolution: like any other science it is predictable. Philos Trans R Soc Lond, 
B, Biol Sci 365(1537):133-145

Dawkins R (1976) The Selfish Gene. Oxford University Press, New York

Dennett D (1995) Darwin's Dangerous Idea. Simon and Schuster, New York

Desjardins E (2009) Historicity in Biology. Master's thesis, University of British Columbia

Desjardins E (2011) Historicity and Experimental Evolution. Biol Philos 26:339-364

Dill K (1999) Polymer principles and protein folding. Protein Sci 8(6):1166-1180

Dryden D, Thomson A, White J (2008), How much of protein sequence space has been explored by life on Earth? J R Soc Interface 5(25):953-956

van Fraassen B (1980) The Scientific Image. Oxford University Press, Oxford

Gould S (1989) Wonderful Life: The Burgess Shale and the Nature of History. Norton, New York

Gould S, Lewontin R (1979) The spandrels of San Marco and the Panglossian paradigm: a critique of

the adaptationist programme. Proc R Soc Lond, B, Biol Sci 205(1161):581-598

Hall, B (2003) Evo-Devo: evolutionary developmental mechanisms. Int J Dev Biol 47(7-8):491-5

Hájek, A (2007) The reference class problem is your problem too. Synthese 156(3):563-585

Huneman P (2010) Topological explanations and robustness in biological sciences. Synthese 177(2):213-245

Hunt B, Ott E (2015) Defining chaos. Chaos 25(9): 097618

Koonin E, Wolf Y, Karev G (2002) The structure of the protein universe and genome evolution. Nature 420(6912):218-223

Ladunga I (1992) Phylogenetic continuum indicates galaxies in the protein universe: Preliminary

results on the natural group structure of proteins. J Mol Evol 4:358-375

Lewis C, Belanger C (2015) The generality of scientific models: a measure theoretic approach.

Synthese 192(1): 269-285.

Lewontin R (1974) The Genetic Basis of Evolutionary Change. Columbia University Press, New York

Maclaurin J, Sterelny K (2008) What is biodiversity? University of Chicago Press, Chicago

Maynard Smith J (1978) Optimization theory in evolution. Annu Rev Ecol Syst 9:31-56

McGhee G (2011) Convergent evolution: limited forms most beautiful. MIT Press, Cambridge

Okamoto T (1988) Analysis of heteromorph ammonoids by differential geometry. Palaeontology

31(1):35-52

Pianka E (1978) Evolutionary Ecology. Harper and Row, New York

Powell R (2009) Contingency and Convergence in Macroevolution: A reply to John Beatty. J Phil

106(7):390-403

Powell R (2012) Convergent evolution and the limits of natural selection. Euro J Phil Sci 2(3):355-373

Powell R, Mariscal C (2015) Convergent evolution as natural experiment: the tape of life

reconsidered. Interface focus 5(6): 20150040

Raup D (1966) Geometric analysis of shell coiling: general problems. J Paleontol 40:1178-1190

Raup D (1967) Geometric analysis of shell coiling: coiling in ammonoids. J Paleontol 41:43-65

Raup D, Michelson A (1965) Theoretical morphology of the coiled shell. Science 147(3663):1294-

1295

Roca-Royes, S (2007) Mind-independence and modal empiricism. Latin Meeting in Analytic

Philosophy. CEUR Workshop proceedings.

Rost B, Liu J, Nair R, Wrzeszczynski K, Ofran Y (2003) Automatic prediction of protein function. Cell Mol Life Sci 60(12):2637-2650

Smith, L (2007) Chaos: A very short introduction. Oxford University Press, Oxford

Strevens, M (2013) Tychomancy: Inferring probability from causal structure. Harvard University Press, Cambridge

Sober E (1988) Reconstructing the past: parsimony, evolution and inference. MIT Press, Cambridge 
Taylor W (2002) A 'periodic table’ for protein structures. Nature 416(6881):657-660

Thomas R, Reif W (1993) The skeleton space: a finite set of organic designs. Evolution 47:341-360

Trinick, J (1994) Titin and nebulin: protein rulers in muscle? Trends Biochem Sci 19(10):405-409

Turner D (2011) Paleontology: A Philosophical Introduction. Cambridge University Press, Cambridge Weisberg M (2004) Qualitative theory and chemical explanation. Philosophy of Science 71(5): 10711081. 